\title{
Progesterone treatment in women with a threatened miscarriage remains controversial after reviewing the available literature! (Mini-commentary on BJOG-19-1550.R1)
}

\author{
Angelo Hooker ${ }^{1}$ \\ ${ }^{1}$ Zaans Medical Center (ZMC)
}

April 28, 2020

Mini-commentary on BJOG-19-1550.R1: Effect of Progestogen for Women with Threatened Miscarriage: A Systematic Review and Meta-analysis

Progesterone treatment in women with a threatened miscarriage remains controversial after reviewing the available literature!

Angelo B. Hooker

Zaans Medical Center (ZMC)

Zaandam 1500 EE Netherlands

Email: hooker.a@zaansmc.nl

Approximately $20 \%$ of pregnant women will experience vaginal bleeding during the first half of their pregnancy, also known as threatened miscarriage (Everett. BMJ 1997;135:32-4). Progesterone has been postulated as a possible treatment option in order to reduce the chance of a miscarriage, but the evidence is limited (Wahabi et al. Cochrane Database Syst Rev 2018;8:CD005943). Whether administration of progesterone could improve live birth has not been assessed yet.

$\mathrm{Li}$ and co-workers conducted a systematic review and meta-analysis (BJOG $2020 \mathrm{xxxx}$ ): 10 trails evaluating the use of progesterone in women with threatened miscarriage were included. Live birth was reported in 6 trails $(\mathrm{n}=4790)$, in $72.9 \%$ in the progesterone group versus $69.7 \%$, relative risk (RR) 1.07 (95\% CI 1.00 - 1.15). There were no differences in the incidence of preterm birth, low birth weight and in congenital abnormalities. Miscarriage was reported in 10 trails $(\mathrm{n}=5180)$, in respectively $18.5 \%$ versus $21.9 \%$, RR 0.73 (95\% CI $0.59-0.92)$.

Although the authors should be complemented for conducting this systematic reviews and meta-analysis, the results should be interpreted cautiously. Nine relatively small trails, with less than 200 cases were included and one trial with more than 4000 women: the results were largely driven by this study. The duration of the progesterone treatment ranged from several days till 16 weeks of gestation, while in two trails it was unclear.

Live birth was reported in 6 studies with difference in baseline characteristics while the risk-of-bias assessment was low in 2, unclear in 1 and high in 3 trails. Furthermore, there were several interacting factors. The type and dose of progesterone varied across the trials. Women with an episode of vaginal bleeding before 24 weeks of gestation were included but when stratified for gestational age at inclusion, the live birth was different: RR 1.04 (95\% CI 1.00 to 1.08) until 12 weeks of gestation compared to RR 1.17 (95\% CI 1.04 to 1.32) between 13-20 weeks. The route of administration also seems to have an effect on live birth rate: 
RR 1.04 (95\% CI 1.00 to 1.08) in the vaginal group compared to RR 1.17 (95\% CI 1.04 to 1.31) in the oral group. The results of the vaginal progesterone group were dominated by one large study.

Notably, the definition of live birth varied: one study used term birth, one birth beyond 22 weeks, one beyond 34 weeks while in 3 trails it was not specified. It was unclear in the studies what the treatment method was in case of repetitive bleeding episodes. The reported diversities and differences lead to clinical heterogeneity.

Despite the reported limitations, $\mathrm{Li}$ and co-authors must be complimented for acknowledging an important limitation in our knowledge and for conducting this clinically relevant trail. Current evidence suggests that administration of progesterone in women with threatened miscarriage may be associated with a higher incidence of live birth and reduction of miscarriage. As suggested by the authors, to confirm the current findings individual participants data meta-analyses and new large randomized trails are needed before introduction in clinical practice.

No disclosures: A completed disclosure of interest form is available to view online as supporting information. 